\title{
Registration of Reconstructed Post Mortem Optical Data with MR Scans of the Same Patient
}

\author{
E. Bardinet ${ }^{1}$, A.C.F. Colchester ${ }^{2}$, A. Roche ${ }^{1}$, Y. Zhu ${ }^{2}, \mathrm{Y} \mathrm{He}^{2}$, S. Ourselin ${ }^{1}$, \\ B. Nailon ${ }^{3}$, S.A. Hojjat ${ }^{2}$, J. Ironside ${ }^{3}$, S. Al-Sarraj ${ }^{4}$, N. Ayache ${ }^{1}$, and \\ J. Wardlaw ${ }^{3}$ \\ 1 INRIA, Epidaure Project, Sophia Antipolis, France \\ 2 KIMHS, University of Kent at Canterbury, UK \\ 3 National Creutzfeldt-Jakob Disease Surveillance Unit, WGH, Edinburgh, UK \\ 4 Institute of Psychiatry, London, UK \\ ebard@sophia.inria.fr
}

\begin{abstract}
We present a method for registration of macroscopic optical images with MR images of the same patient. This forms a key part of a series of procedures to allow post mortem findings to be accurately registered with MR images, and more generally provides a method for 3D mapping of the distribution of pathological changes throughout the brain. The first stage of the method involves a $3 \mathrm{D}$ reconstruction of $2 \mathrm{D}$ brain slices and was presented in a previous paper [2]. In the current paper, we focus on the registration of the reconstructed volume with corresponding MR images.
\end{abstract}

\section{Introduction}

The correlation of pathological findings with abnormalities on MR images is important in many neurological conditions. In Creutzfeldt-Jakob disease (CJD), MR signal changes have been observed in certain locations, for example (in variant CJD) in the posterior thalamus (pulvinar) [10], but pathological changes are known to be more widespread, and it is likely that grey matter signal abnormality occur in a much wider distribution. Accurate 3 -D registration of $\mathrm{MR}$ and large field-of-view pathology images could help to clarify the distribution and mechanism of MR signal changes.

In a previous publication 2], we described work on the 3D reconstruction of macroscopic optical images of brain slices into a a 3D coordinate frame. During routine clinical neuropathology, such slices are cut by hand, the slice thickness being controlled by a simple guide on which a knife is rested while cutting. 3D reconstruction of the macroscopic optical data would be easier to accomplish if thin sections could be obtained from a macrocryotome [8]. However cryosections have to be cut from fresh frozen ("unfixed") brain which is highly infectious in CJD. The apparatus would thus have to be retained for use on CJD brains only, and the subsequent specialised processing of large thin sections would also 
require dedicated equipment which could not be used for any other types of patients. The precaution needed would be complex, very expensive, and probably prohibitive. Furthermore, the large cryosections could not be proceed in the way needed for routine diagnostic neuropathology. Therefore, we have devoted considerable effort into developing methodology for reconstruction of macroscopical optical brain slice images which can be incorporated into a routine clinical environment [2].

In the present paper, we take such $3 \mathrm{D}$ reconstructions of optical brain slice data and show that these can be successfully registered with MR scans of the same patient. This is a further major step towards our goal of relating microscopic pathology data to the exactly corresponding locations in MR scans.

\section{Methods}

The left hemisphere of a formalin-fixed post mortem brain was examined for this preliminary study. First, several MR images were acquired (Proton-Density, T1, T2, FLAIR) with the brain suspended in a water-filled container which reduced brain distortion due to gravity and dampened oscillations transmitted from the scanner. The brain was aligned in the scanner following the normal clinical procedure as far as possible, but it was not expected that the MR slice planes would correspond exactly to the physical brain slices which were to be cut later. Fig. [1 shows three orthogonal views of the Proton-Density image. After brain fixation, the pathologist cut 14 coronal slices $12 \mathrm{~mm}$ thick through the brain. The anterior $(\mathrm{A})$ and posterior $(\mathrm{P})$ faces of each slice were photographed. A perspex jig was constructed to hold each slice during photography [2]. Accurate circular fiducial marks were machined at each corner. Slices were numbered from front to back (Fig. 2 shows the images from slices 2, 3 and 4). Section 2.1
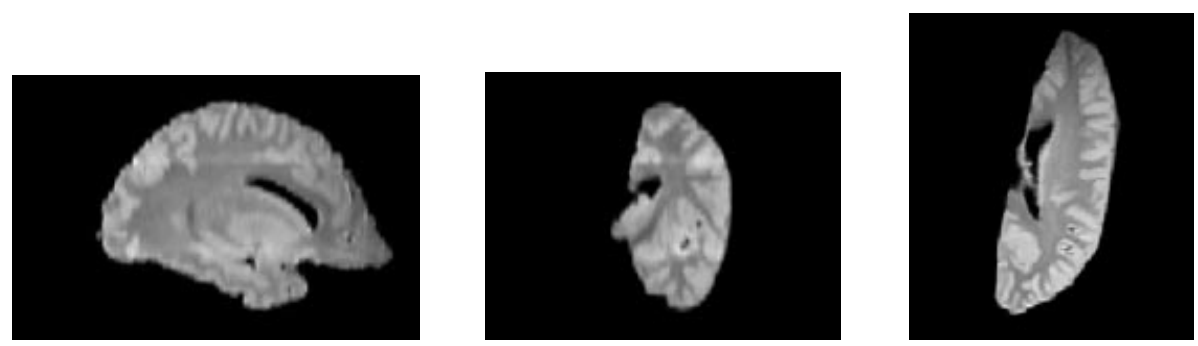

Fig. 1. Post mortem MR Proton-Density image. From left to right: sagittal, coronal and axial views.

briefly summarizes the method used to realign the optical sections, allowing the reconstruction of a $3 \mathrm{D}$ block. Details are found in [2]. In the present paper, we restricted ourselves to rigid slice realignment when reconstructing 3-D optical data sets. Although more complex 2-D transformations between adjacent slices 

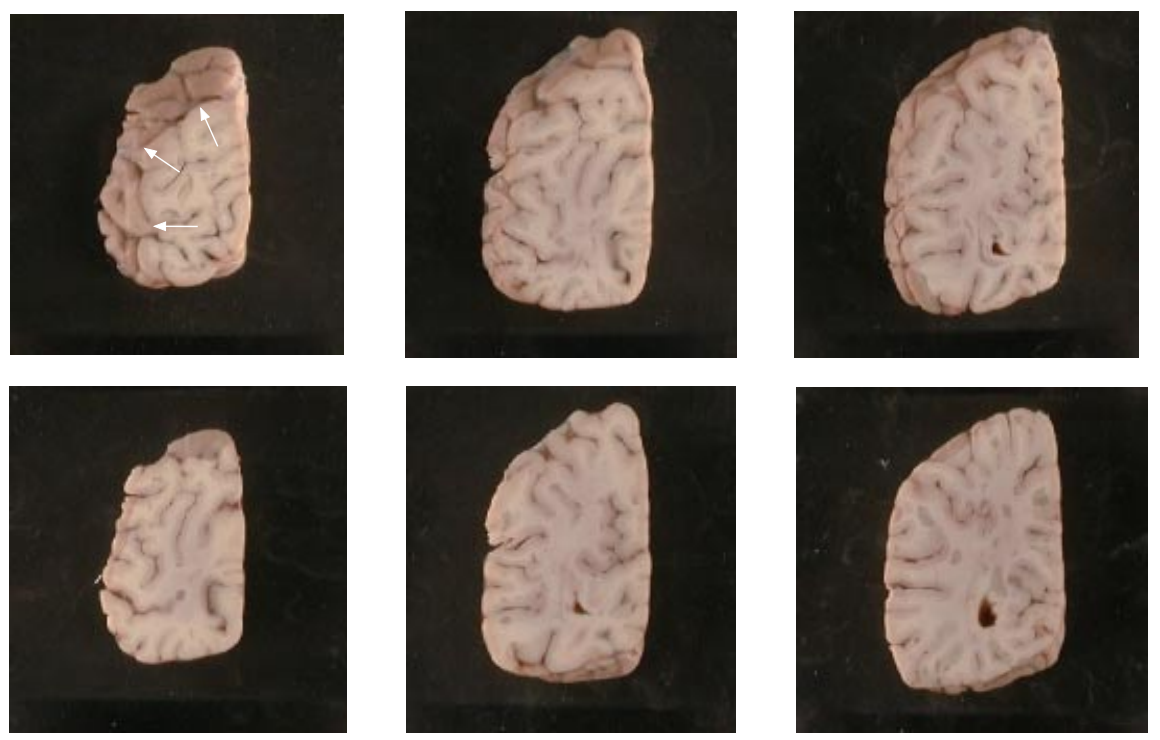

Fig. 2. Macroscopic optical images of three brain slices. Top row: anterior images (A2, A3, A4); bottom row: corresponding posterior images of the same slice (P2, P3, P4). In image A2, arrows show the boundary between the cut surface of the slice and the oblique (non-cut) surface forming the side of the slice.

can be estimated from the optical data alone 2], the lack of 3D prior information makes it hard to evaluate accuracy and deformations in the $\mathrm{z}$ direction cannot be retrieved. The geometric distortions in the optical data compared to the MR data can in theory be fully recovered during $3 \mathrm{D} / 3 \mathrm{D}$ registration between the reconstructed block and an MR image of the same patient. Schormann et al. [7] previously proposed a method based on a series of slice-by-slice block-matching strategies to estimate a global 3-D affine transformation, but they assumed that out-of-plane motion could be neglected, which is not valid for our application. We thus propose another $3 \mathrm{D}$ multimodal affine registration method in section 2.2.

\subsection{D Reconstruction of Macroscopic Optical Brain Slice Images}

To reconstruct the original 3D relationships, two different types of registration had to be computed. The first was to register the anterior and posterior sides of each slice, i.e. within-slice registration. The second was to register the images of surfaces on either side of the cut which separates two adjacent slices, i.e. betweenslice registration. Finally by propagating the $2 \mathrm{D}$ registrations through the volume and using the known slice thickness, the $3 \mathrm{D}$ volume was reconstructed.

Within-Slice Co-registration Using Artificial Landmarks The cut surfaces on each side of a slice are separated in the direction perpendicular to the 
faces by several millimetres. We therefore cannot rely on sufficient similarity between image features to match them correctly. Instead, fiducials on the jig were detected automatically and point-based rigid registration computed.

Between-Slice Intensity-Based Registration The surfaces on either side of a single cut were derived from the cleavage of a single tissue plane and natural features on the two cut surfaces should correspond exactly to each other. Although, distortions occur during cutting and during later movement of a slice, rigid co-registration using a robust block matching algorithm provided a good match [4].

3D Reconstruction by Propagation of 2D Registrations through the Volume The known slice thickness was used to calculate the antero-posterior coordinate ( $z$ value) of each image. The alternating within-slice and betweenslice rigid registrations were propagated from slice 7 (approximately the largest slice) near the middle of the volume, forwards and backwards through smaller slices in the volume, to allow each image to be transformed in $x$ and $y$.

\subsection{Registration between Reconstructed Optical Data and MR Images of the Same Patient}

3D Rigid Registration: Surface-Based Method We first perform a rigid Iterative Closest Point (ICP) registration [1]11] between the brain surfaces extracted from both the MR and optical volumes. Notice that the MR surface has a much higher resolution along the $z$-axis than the reconstructed optical block surface (3 mm vs $12 \mathrm{~mm}$ thickness).

In the optical images, the surface extracted automatically was in fact the outer silhouette rather than the boundary of the cut surface (see Fig. 2, slice A2). This causes the ICP algorithm to match surface points from the MR image with some non-corresponding points from the optical block. Therefore, one sees that the accuracy of a surface-based registration is intrinsically limited by the amount of visible non-cut surfaces. Nevertheless, it provides a fair starting estimate for the subsequent intensity-based registration, a method generally quite dependent on good initialisation.

3D Affine Registration: Intensity-Based Method In order to improve the ICP result and to achieve affine registration, we use an intensity-based approach which enables us to match homologous regions rather than contours. Starting from the rigid estimate provided by the ICP, we search for the affine transformation that maximises a similarity measure between the MR image and the reconstructed optical block.

A robust variant of the correlation ratio based on the Geman-McClure scale estimator was used as the similarity measure, as in our previous work 6]. The assumption underlying the correlation ratio is that there exists an unknown mapping from the MR intensities to the reconstructed optical block intensities. This 
assumption actually does not hold in the whole image overlap whenever regions with small intensity variance in the MR image are expected to match regions with high intensity variance in the optical block. This will typically occur here because MR background voxels are expected to match non-cut surfaces voxels. The use of a robust correlation measure prevents the registration from being biased by such outliers. Other similarity measures such as mutual information $[3,9, ?]$ could also be used. However, we found the correlation ratio measures to be generally easier to optimise as they yield fewer local maxima [5].

\section{Results and Discussion}
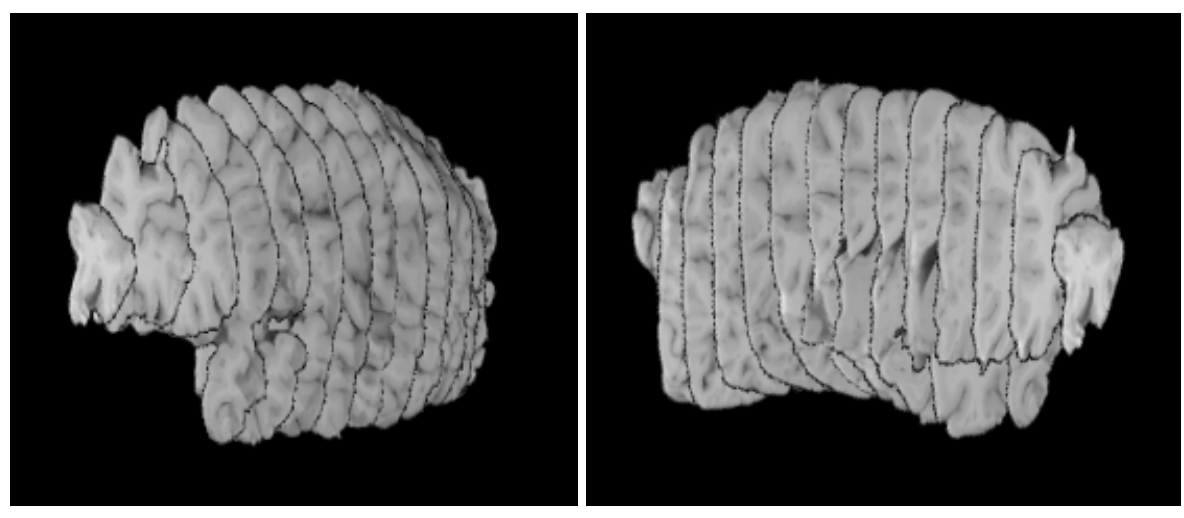

Fig. 3. 3D reconstruction of macroscopic optical brain slice images from a left hemisphere (only images of the anterior faces are shown). Left: antero-lateral view. Right: antero-medial view.

We present in Fig. 3 the 3D reconstructed block obtained after rigid realignment of the macroscopic optical brain slices. The result of the 3D affine registration between the reconstructed block and the MR Proton-Density image is shown in two different ways in Figures 4 and 5. Fig. 4 presents four different optical slices overlaid with brain contours extracted from the MR image, respectively: before registration, after ICP rigid registration, and after correlation ratio-based affine registration. In order to better assess the effect of registration in 3D, we show in Fig. 5 three different perspective views of the MR and optical volumes, respectively before and after registration.

Our results show that the methods we have developed do indeed allow successful registration between images of physically-cut post-mortem slices and MR data from the same post-mortem brain acquired before cutting. In the present paper, evaluation of the success of registration has been based on visual inspection of $2 \mathrm{D}$ slices through the volume data. It is relatively easy to judge the 

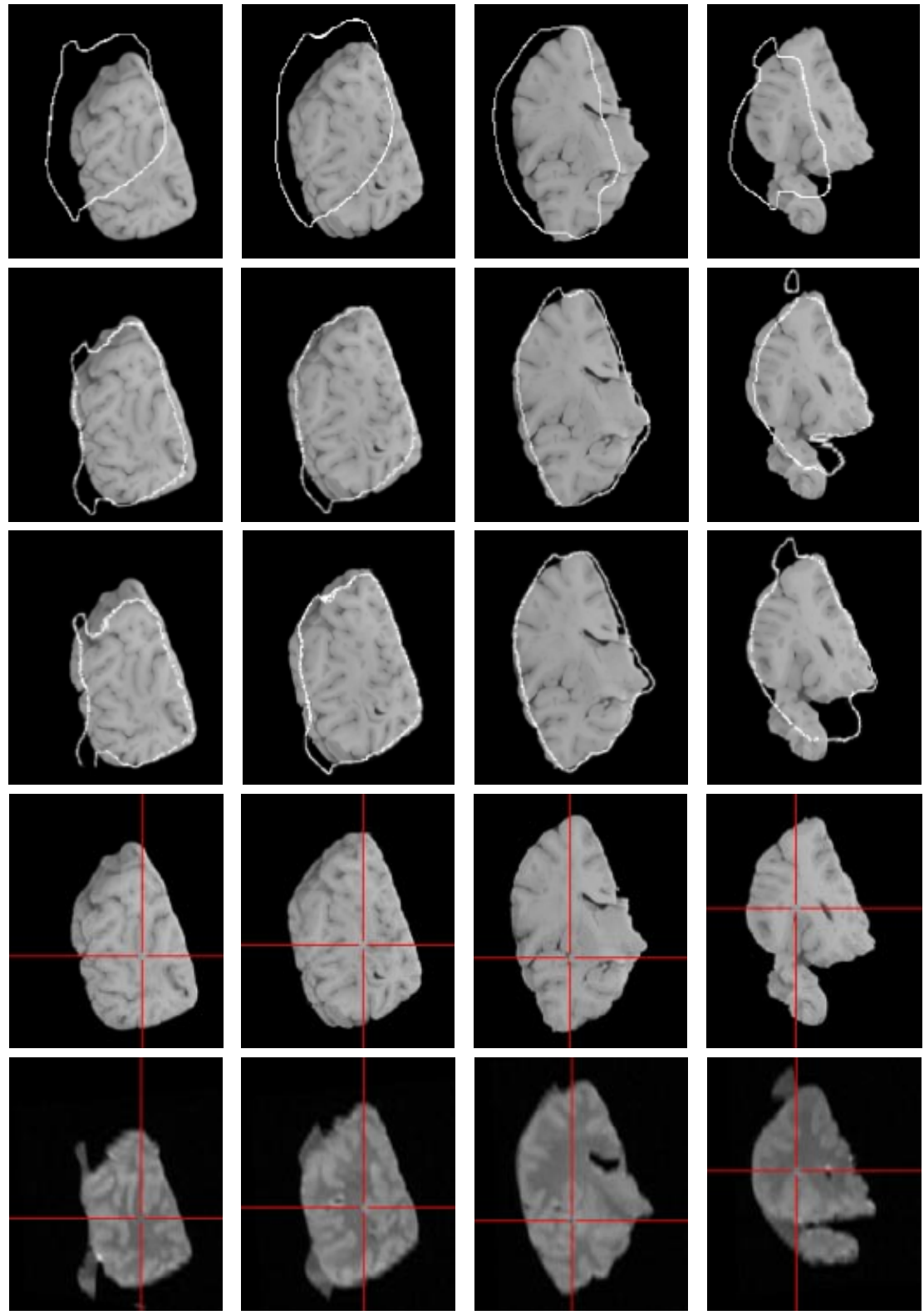

Fig. 4. Rows 1, 2, 3: contours of post mortem MR image (outer surface of brain hemisphere) superimposed on optical slices; row 1: before matching; row 2: after contour-based rigid matching; row 3: after intensity-based affine matching. Rows 4, 5: optical and MR slices after intensity-based affine registration, with cursor superimposed to help visual inspection of the correspondence between visible features in the two modalities. Columns correspond to different slices in the volumes. 
correspondence of outlines of $2 \mathrm{D}$ sections through the registered $3 \mathrm{D}$ data. However, the optical data are digital photographs of a physically sliced brain; in addition to the cut surface, the non-cut surface may be visible adjacent to the cut surface in the same photograph, if the non-cut surface is at an appropriate angle to the camera viewpoint so that it is not occluded. Therefore, evaluation by visual inspection of the quality of the matching after registration needs to take account of the fact that the boundary of a thin MR section should correspond to the boundary of the cut surface in the optical data set, which is not necessarily the same as the outer silhouette of the optical image. Inspection of the registered outlines in Fig. 4 confirms that a good result has been achieved. Judicious use of outlier rejection in the final stage of $3 \mathrm{D}$ intensity-based affine registration has ensured that the match has been successful. Inspection of the correspondence between visible features in the two modalities in Figures 4 and 5 confirms the good matching even in the presence of a substantial amount of non-cut surface in some optical images.
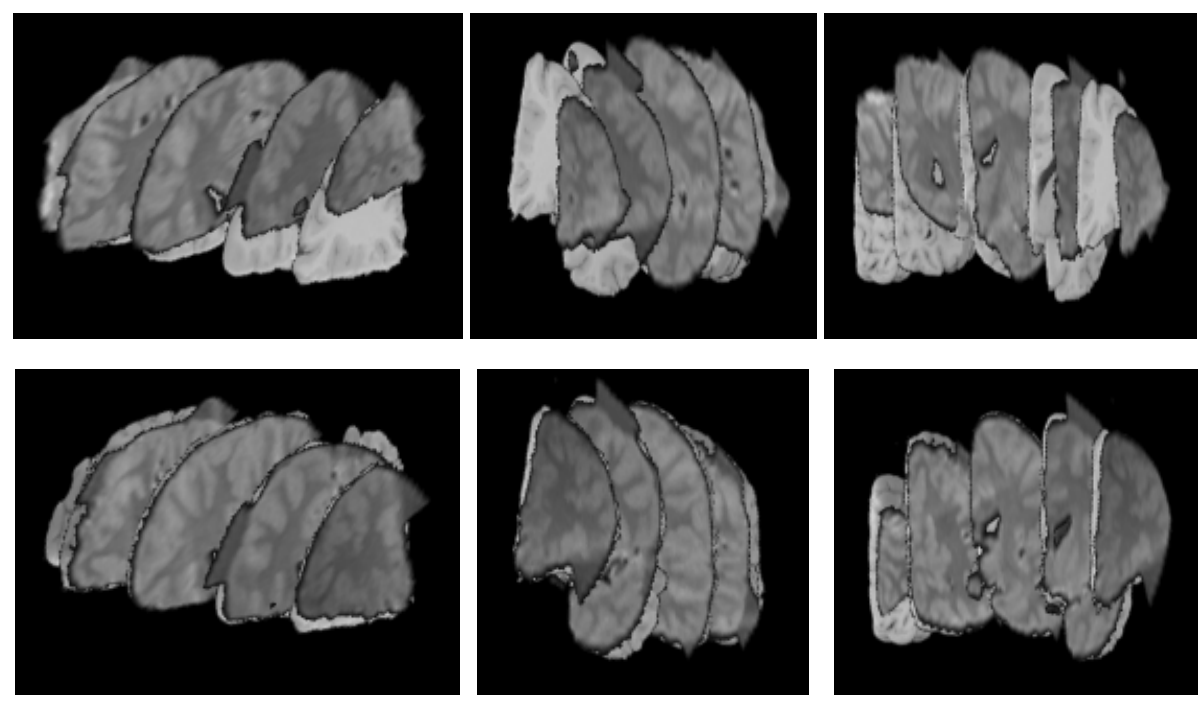

Fig. 5. Perspective views of five selected corresponding slices of the post mortem $M R$ image and the reconstructed macroscopical volume. First row: before registration. Second row: after 3D affine registration. Left column: antero-superior view. Middle column: antero-lateral view. Right column: antero-medial view.

When brain slices are cut by the pathologist, new distortions are inevitably introduced. Apart from smooth distortions, which may be visualised as those occurring when a loaf of bread is cut, there are additional problems when separated fragments of brain can move independently of each other. Thus, if a coronal section is cut through the frontal lobe and also the temporal pole, the temporal fragment may be completely disconnected from the remainder of the hemisphere, 
and it is impossible to avoid some degree of independent movement between the two fragments. In the current work, the aim was to register the majority of the slice correctly, leaving fragments which had moved independently as outliers (i.e. not registered at this stage). Future refinements will include methodology to allow free-form movement of discrete parts relative to each other. Within parts, affine deformation is likely to remain a good approximation to the real deformations in continuous blocks of fixed brain tissue.

Just as there are deformations between post-mortem MR of a fixed brain hemisphere and reconstructed 3D volumes from optical images of brain slices, so also there are deformations between in vivo MR and subsequent MR of postmortem brains. However, if care is taken to suspend the brain during fixation in formalin, we believe that most of the latter deformations are less complex than those between post-mortem MR and post-mortem optical data, and we expect to be able to apply the same basic approach to direct registration between in vivo MR and post mortem optical data.

Work is currently in progress to register thin microscopic sections, on which quantitative pathology is performed, with the macroscopic optical brain slices. This will complete the sequence of transformations necessary for mapping microscopic data into in vivo MR scans of the same patient.

\section{Conclusion}

We have developed a method for registration of macroscopic optical images with MR images of the same patient. The first stage of the method involved a 3D reconstruction of $2 \mathrm{D}$ brain slice images, and this reconstruction is then registered with the MR data. Our preliminary evaluation indicates that an excellent registration can be achieved. This is a key part of a series of procedures to allow histological findings to be accurately registered with in vivo MR images.

\section{Acknowledgements}

This work was supported by the EU-funded QAMRIC project BMH 4-98-6048 (Quantitative Analysis of MR Scans in Creutzfeldt-Jakob Disease, http://wwwsop.inria.fr/epidaure/qamric). The imaging was performed at the SHEFC Brain Imaging Research Centre for Scotland. We thank Hervé Delingette for the visualisation software.

\section{References}

1. P.J. Besl and N.D. McKay. A Method for Registration of 3-D Shapes. IEEE Transactions on Pattern Analysis and Machine Intelligence, 14(2):239-256, 1992.

2. A.C.F. Colchester, S. Ourselin, Y. Zhu, E. Bardinet, Y. He, A. Roche, S. AlSarraj, B. Nailon, J. Ironside, and N. Ayache. 3-D Reconstruction of Macroscopic Optical Brain Slice Images. In S.L. Delp, A.M. DiGioia, and B. Jaramaz, editors, Proceedings of MICCAI'00, volume 1935 of LNCS. Springer, 2000. 
3. F. Maes, A. Collignon, D. Vandermeulen, G. Marchal, and P. Suetens. Multimodality Image Registration by Maximization of Mutual Information. IEEE Transactions on Medical Imaging, 16(2):187-198, 1997.

4. S. Ourselin, A. Roche, G. Subsol, X. Pennec, and N. Ayache. Reconstructing a 3D Structure from Serial Histological Sections. Image and Vision Computing, 19(1-2):25-31, January 2001.

5. A. Roche, G. Malandain, and N. Ayache. Unifying Maximum Likelihood Approaches in Medical Image Registration. International Journal of Imaging Systems and Technology, 11:71-80, 2000.

6. A. Roche, X. Pennec, M. Rudolph, D. P. Auer, G. Malandain, S. Ourselin, L. M. Auer, and N. Ayache. Generalized Correlation Ratio for Rigid Registration of 3D Ultrasound with MR Images. In S.L. Delp, A.M. DiGioia, and B. Jaramaz, editors, Proceedings of Medical Imaging Computing And Computer-Assisted Intervention (MICCAI'00), volume 1935 of LNCS, pages 567-577. Springer, 2000.

7. T. Schormann, M. Von Matthey, A. Dabringhaus, and K. Zilles. Alignment of 3-D Brain Data Sets Originating From MR and Histology. Bioimaging, 1:119-128, 1993.

8. V. Spitzer, M.J. Ackerman, A.L. Scherzinger, and D. Whitlock. The visible human male: a technical report. Journal of American Medical Informatics Association, 3(2):118-130, 1996.

9. P. Viola. Alignment by Maximisation of Mutual Information. International Journal of Computer Vision, 24(2):137-154, 1997.

10. M. Zeidler, R.J. Sellar, D.A. Collie, R. Knight, G. Stewart, M.A. Macleod, J.W. Ironside, S. Cousens, A.C.F. Colchester, D.M. Hadley, and R.G. Will. The pulvinar sign on magnetic resonance imaging in variant Creutzfeldt-Jakob disease. Lancet, 355(9213):1412-1418, April 2000.

11. Z. Zhang. Iterative Point Matching for Registration of Free-Form Curves and Surfaces. International Journal of Computer Vision, 13(2):119-152, 1994. 